\title{
Teneur en ADN de la larve de Dicentrarchus labrax: évolution ontogénétique et effet de la privation de nourriture
}

\author{
Jean-Pierre Bergeron ${ }^{(1)}$ et Jeannine Person-Le Ruyet ${ }^{(2)}$ \\ (1) Laboratoire Écologie Halieutique, Direction des Ressources Vivantes, \\ IFREMLR, Centre de Nantes, B.P. 21105, F- 44311 Nanles cedex 03, France. \\ E-mail :Jean.Pierre.Bergeron@ifremer.fr \\ (2) Laboratoire Physiologie des Poissons, Direction des Ressources Vivantes, \\ IFREMFR, Centre de Brest, B.P. 70, F-29280 Plouzané, France.
}

Reçu le 17 septembre 1996 ; accepté le $1^{\text {er }}$ avril 1997.

Bergeron J. P., J. Person-Le Ruyet. Aquat. Living Resour., 1997, 10, 247-250.

Relative DNA content of larval sea bass Dicentrarchus labrax: ontogenetic changes and effect of starvation.

Abstract

Sea bass Dicentrarchus labrax larvae were hatched and reared for 34 days in the laboratory to test a recent biochemical index (DNA/C) of larval fish nutritional condition. Variations in DNA/C ratio values were monitored during the whole larval ontogenesis of fed individuals and the effect of starvation was assessed at three developmental stages. Three main periods in the relative DNA content variations were determined in close connection with growth patterns during larval ontogenesis.

Keywords : Dicentrarchus labrax, DNA/C, nutritional condition, starvation, growth, biochemical index.

\section{INTRODUCTION}

Soupçonnée d'être parfois la cause de fortes mortalités dans le milieu naturel, l'inanition des stades larvaires de poissons marins peut être à l'origine de la variabilité du recrutement de certaines populations et la recherche de méthodes susceptibles de la caractériser demeure actuellement l'objet de nombreux travaux (Ferron et Leggett, 1994). Un indice biochimique simple, fondé sur la teneur relative en $\mathrm{ADN}$ de la larve, a été proposé (Bergeron et al., 1991), puis techniquement amélioré (Bergeron et al., 1997) et testé expérimentalement chez la sole (Solea solea). L'ontogenèse larvaire de cette espèce présente une phase très typique des Pleuronectiformes : à symétrie bilatérale à l'éclo- sion, la larve subit, lors de sa métamorphose, de profonds remaniements anatomiques et morphologiques qui lui donneront progressivement sa forme définitive de « poisson plat ». La teneur en ADN présente remarquablement deux niveaux moyens différents et caractéristiques respectivement de la larve à symétrie bilatérale d'une part et en cours de métamorphose d'autre part (Bergeron et al. 1997). Ces deux niveaux permettent de définir chez la larve témoin nourrie des seuils quantitatifs au-delà desquels l'inanition peut être déterminée sans ambiguïté (Bergeron et al., 1991). Quelle est la portée de ce modèle de variations de l'indice ? Est-il particulier à la sole ou plus général ? Et notamment comment évolue la teneur en ADN de larves d'espèces dont la métamorphose ne s'accompa- 
gne pas de modifications morphologiques aussi spectaculaires ? Une première approche d'éléments de réponse à ces questions a motivé le travail expérimental dont les résultats sont présentés ici. Il était essentiel de n'avoir pas à se heurter d'emblée à des problèmes de technique d'élevage, c'est pourquoi nous avons choisi le bar (Dicentrarchus labrax), dont les conditions d'élevage larvaire au laboratoire sont bien maîtrisées, comme modèle de «poisson rond ». L'évolution de la teneur en $A D N$, exprimée par rapport au poids de carbone (C), a été suivie chez des larves témoins nourries dans des conditions standard et privées de nourriture à trois stades de leur développement.

\section{MATÉRIEL ET MÉTHODES}

L'élevage expérimental a été mené au Centre de Brest de l'IFREMER, dans les installations du Département Ressources Aquacoles spécialement affectées à l'élevage de larves de poissons. Les larves sont issues d'une ponte spontanée, au mois de mai, de géniteurs en captivité. Le lendemain de l'éclosion (J1), elles sont mises en élevage à $15^{\circ} \mathrm{C}$ en bassins de $250 \mathrm{l}$ à fond hémisphérique et parois noires et sont maintenues en milieu non renouvelé et à l'obscurité jusqu'à J4 (ouverture de la bouche). A partir de J4, les bassins sont alimentés en eau de mer filtrée thermorégulée (température moyenne de $19 \pm 1{ }^{\circ} \mathrm{C}$ ) et l'intensité lumineuse est augmentée progressivement. Elle est stabilisée à 1500 lux en surface pour la plage la plus éclairée à partir de J20, la photophase étant $18 \mathrm{~h}$ par cycle de $24 \mathrm{~h}$. Le renouvellement d'eau est de $10 \% . \mathrm{h}^{-1} \mathrm{de} \mathrm{J} 4 \mathrm{à} \mathrm{J} 11$ et de $30 \%$ par la suite, le niveau d'oxygène étant au-dessus de $90 \%$ de la saturation. La salinité est de $30 \pm 1$. La charge initiale est de 33 larves. $1^{-1}$, elle décroît progressivement du fait de prélèvements réguliers de larves pour échantillonnage ( 3500 larves de $\mathrm{J} 1$ à J30). Les larves des lots témoins sont nourries une fois par jour à partir de proies d'élevage, à savoir : rotifères (Brachionus plicatilis) de J4 à J16, nauplii d'Artemia juste éclos de J11 à $\mathrm{J} 27$ (souche San Francisco Bay Brand), metanauplii de 2 jours d'Artemia de J22 à J34.

L'expérience consiste à suivre les lots témoins pendant le développement larvaire jusqu'à la métamorphose et à priver les larves de nourriture dès l'ouverture de la bouche à J4 et, après avoir été nourries dans les conditions décrites ci-dessus, de façon différée à $\mathrm{J} 15$ et J28. Chaque traitement est appliqué simultanément à deux lots de larves élevées dans des bacs séparés, l'ensemble comprend donc initialement 8 bacs. Notons qu'un incident technique est survenu dès le début de l'expérience dans un des bacs, ce qui nous a conduit à ne conserver qu'un seul lot de larves pour observer l'effet de la mise à jeun à J28. De plus, un autre incident à $\mathrm{J} 10$, sur l'un des deux lots privés de nourriture dès l'ouverture de la bouche, nous a retiré deux valeurs pour $\mathrm{J} 12$ et $\mathrm{J} 14$ (voir triangles ouverts, Fig. 1, 2).
Les modes de prélèvement et de préparation des échantillons, les méthodes d'analyse utilisées sont décrits dans Bergeron et al. (1997). Il convient juste de préciser ici que l'effectif de chaque échantillon est de 50 individus et de rappeler que le dosage des acides nucléiques est réalisé selon la méthode fluorimétrique au bromhydrate d'ethidium initialement proposée par Le Pecq et Paoletti (1966), modifiée par Karsten et Wollenberger $(1972,1977)$. Les mesures de carbone ont été effectuées sur le même homogénat à l'aide d'un analyseur CHNS/O plus performant que précédemment (Perkin Elmer 2 400).

\section{RÉSLILTATS}

La courbe de croissance des larves témoins nourries, en termes d'évolution de leur poids de carbone, montre trois phases très distinctes (Fig. 1). Pendant la première dizaine de jours après l'éclosion (jusqu'à J1012 ), le poids reste stable, voire tend à légèrement diminuer, ce qui traduit la résorption progressive de la vésicule vitelline. Durant les dix jours suivants apparaît une forte croissance qui se déclenche soudain (à partir de $\mathrm{J} 10$ pour un lot, de $\mathrm{J} 12$ pour l'autre, les raisons de ce décalage n'ont pas pu être déterminées) et se conforme à une loi exponentielle. Enfin entre J20-22 et la fin de l'expérience (J34) la croissance se poursuit toujours selon une fonction exponentielle mais de pente plus faible.

L'effet de privation totale de nourriture n'est pas perceptible au niveau pondéral tant que se résorbe le vitellus (Fig. 1) et apparaît dès que se déclenche la première phase exponentielle de la croissance des individus nourris. En revanche, l'effet des mises à jeun différées se manifeste en deux jours par un arrêt de la croissance, puis par une claire diminution du poids (Fig. 1).

L'évolution de la teneur en ADN (ADN/C) des larves témoins nourries présente également trois périodes caractéristiques (Fig. 2). L'indice augmente très régulièrement après l'éclosion pour atteindre des valeurs comprises entre 70 et $80 \mu \mathrm{g} \cdot \mathrm{mg}^{-1}$ vers J10-12. Puis apparaissent deux diminutions successives très abruptes : la première entre $\mathrm{J} 12$ et $\mathrm{J} 16$, la plus forte, conduit à un plateau vers 45-50 jusqu'à J22 . On peut remarquer ici que le décalage d'approximativement deux jours, mentionné précédemment à propos du début de la croissance exponentielle de chacun des deux lots de larves témoins (Fig. 1), se reflète très précisément au niveau des valeurs d'ADN/C relevées à $\mathrm{J} 14$ (Fig. 2) et que ce brusque changement correspond exactement à la résorption totale du globule lipidique, vestige des réserves endogènes sur le point de s'épuiser (à J12 pour le premier lot, à $J 14$ pour le second). A ce stade, les larves mesurent entre 5,5 et $6,1 \mathrm{~mm}$, ce qui correspond à la taille à laquelle Barnabé et al. (1976) situent ce phénomène. Une seconde diminution du rapport ADN/C survient entre $\mathrm{J} 22$ et $\mathrm{J} 24$ et un nouveau plateau 


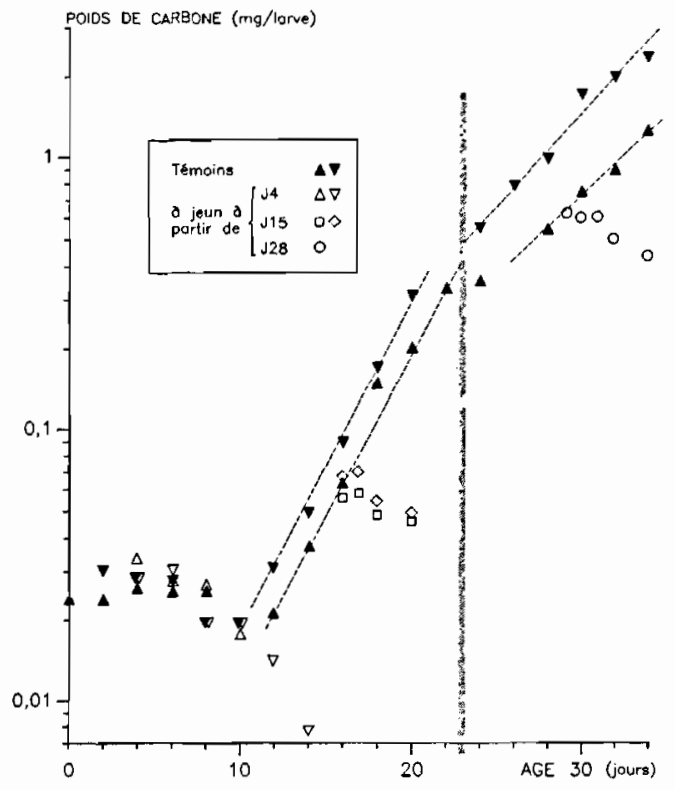

Figure 1. - Croissance pondérale (en $\mathrm{mg}$ de carbone) de la larve de bar, Dicentrarchus lahrax, au cours de son ontogenèse (âge exprimé en nombre de jours après l'éclosion) et effet de la privation de nourriture à différents stades de développement. Un repère ombré à J23 indique le début de la flexion de la notochorde.

Ontogenetic growth (in mg carbon) of larval sea bass Dicentrarchus labrax (age in days after hatching) and effect of starvation (open symbols) at different developmental stages. A shaded mark on D23 indicates the beginning of notochord flexion.

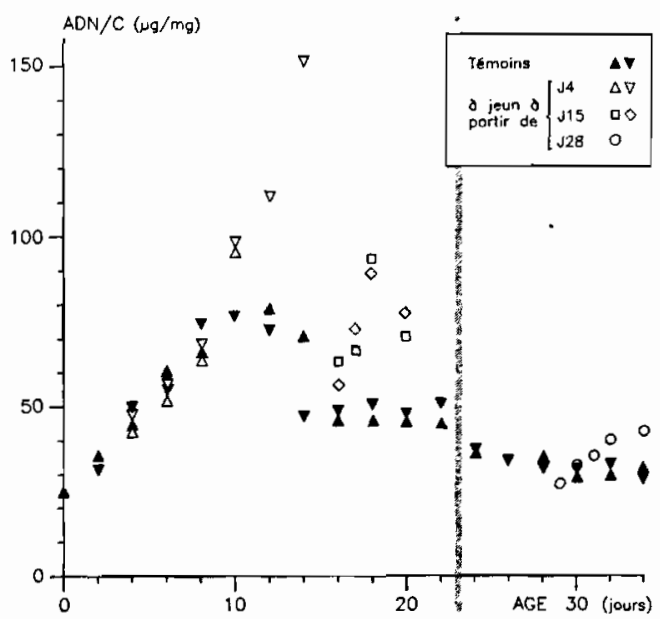

Figure 2. - Évolution de la teneur en ADN (en $\mu \mathrm{g} \cdot \mathrm{mg}^{-1}$ de carbone) de la larve de bar au cours de son ontogenèse (âge exprimé en nombre de jours après l'éclosion) et effet de la privation de nourriture à différents stades de développement. Un repère ombré à $\mathrm{J} 23$ indique le début de la flexion de la notochorde.

Ontogenetic changes in the DNA content (in $\mu \mathrm{g} \cdot \mathrm{mg}^{-1}$ carbon) of larval sea bass (age in days after hatching) and effect of starvation (open symbols) at different developmental stages. A shaded mark on D23 indicates the beginning of notochord flexion. s'établit entre 30 et 35 (Fig. 2). Ici encore notons la remarquable coïncidence des brusques changements des valeurs d'ADN/C (Fig. 2) et de la vitesse de croissance (Fig. 1) qui s'avèrent correspondre au début de la flexion de la notochorde.

Chez les larves privées de nourriture dès l'ouverture de la bouche ( $\mathrm{J} 4$ à $\left.19^{\circ} \mathrm{C}\right)$, l'augmentation progressive des valeurs de la teneur en ADN suit exactement celle des témoins nourris jusqu'à J8 (Fig. 2). A J10 apparaît l'effet du jeûne par une augmentation de l'ordre de 25$30 \%$, nettement renforcée ensuite à $\mathrm{J} 12$, puis $\mathrm{J} 14$. Une mise à jeun différée à $\mathrm{J} 15$ induit un accroissement plus sensible et rapide, proche de $100 \%$ en trois jours (Fig. 2). En revanche, une mise à jeun plus tardive à J28 ne se révèle que discrètement au bout de 4 jours, plus distinctement deux jours plus tard (30-35\% d'augmentation' à J34).

\section{DISCUSSION ET CONCLUSION}

L'ontogenèse de la larve de bar se subdivise très clairement, tant du point de vue de la croissance pondérale que de celui de l'évolution de la teneur en ADN, en trois principales phases. Déjà Gutiérrez et MoralesNin (1986) avaient montré que de telles subdivisions étaient décelables à l'examen des accroissements journaliers d'otolithes de larves de bar, bien que les températures de leur élevage expérimental fussent fluctuantes ; plus récemment, Regner et Dulčić (1994) ont brillamment confirmé, en mettant en relation les taux de croissance en longueur de larves avec ceux enregistrés par les otolithes, que des variations intrinsèques de croissance apparaissent spécifiques des trois principales périodes du développement larvaire présentées ici. L'évolution de la croissance à J22-24 (Fig. 1) appelle un commentaire : à ce stade de la morphogenèse survient un événement marquant, le début de la flexion de la notochorde, que l'on trouvera décrit et bien illustré dans Barnabé et al. (1976); il s'y situe à un âge plus avancé, environ J30, ce qui s'explique parfaitement par la différence de croissance induite par l'écart de température $\left(15^{\circ} \mathrm{C}\right.$ au lieu de $19^{\circ} \mathrm{C}$ dans notre étude). Ce stade précis est remarquable à plus d'un titre : la nageoire primordiale régresse très fortement alors que simultanément la masse musculaire devient plus volumineuse (Barnabé et al., 1976). Comme le souligne Blaxter (1988), la flexion de la notochorde s'accompagne d'un rapide changement de la forme du corps, dont notamment le développement de la nageoire caudale : il s'agit d'un événement essentiel dans la vie de la larve car dès lors la capacité locomotrice s'accrô̂t de façon considérable (phénomène précisément noté chez le bar par Barnabé et al., 1976). Nos résultats confirment que des modifications du métabolisme surviennent également, se traduisant par un ralentissement de la croissance (Fig. 1) et une diminution corrélative de la teneur en ADN (Fig. 2), révélatrice de l'hypertrophie cellulaire caractéristique du développement des muscles locomoteurs (Goss, 1966). Le schéma global 
de développement de la larve de bar se trouve ainsi tout à fait analogue à ceux d'autres espèces pour lesquelles Kendall et al. (1984) proposent les subdivisions en «yolk-sac», "preflexion» et «flexion»: nos résultats semblent en confirmer le bien-fondé puisqu'à ces stades correspondent des valeurs caractéristiques des teneurs en ADN. Ajoutons enfin que l'ordonnance séquentielie évidente de celles-ci apporte un argument d'ordre métabolique à l'appui de la théorie de l'ontogenèse saltatoire (Balon, 1984).

L'augmentation de la teneur en ADN (ADN/C) consécutive à la privation de nourriture mérite d'être brièvement comparée à celle observée chez la sole (Bergeron et al., 1991) : comme chez celle-ci, la rapidité et l'amplitude de la réponse décroissent à mesure

\section{RÉFÉRENCES}

Balon E.K. 1984. Reflections on some decisive events in the early life of fishes. Trans. Am. Fish. Soc. 113, 178-185.

Barnabé G., F. Boulineau-Coatanea, F. René 1976. Chronologie de la morphogénèse chez le loup ou bar Dicentrarchus labrax (L.) (Pisces, Serranidae) obtenu par reproduction artificielle. Aquaculture 8, 351-363.

Bergeron J.P., M. Boulhic, R. Galois 1991. Effet de la privation de nourriture sur la teneur en ADN de la larve de sole (Solea solea L.). ICES J. Mar. Sci. 48, 127-134.

Bergeron J.P., J. Person-Le Ruyet, C. Koutsikopoulos 1997. Use of carbon rather than dry weight to assess the DNA content and nutritional condition index of sole larvae. ICES J. Mar. Sci. 54, 148-151.

Blaxter J.H.S. 1988. Pattern and variety in development. In : Fish physiology, vol, 11 A. W.S. Hoar, D.J. Randall eds. Academic Press, London, 1-58.

Ferron A., W.C. Leggett 1994. An appraisal of condition measures for marine fish larvae. Adv. Mar. Biol. 30, 217-303.

Goss R.J. 1966. Hypertrophy versus hyperplasia. Science $153,1615-1620$. que se développent les larves et la variation de l'indice devient sensiblement moins perceptible quand la privation de nourriture survient à J28. Pour le jeûne précoce, un certain temps de latence, probablement dû à la présence de la vésicule vitelline (on peut d'ailleurs noter que les larves se nourrissent très peu pendant les tout premiers jours), précède l'accroissement des valeurs de l'indice, qui dans ce cas apparaît très fort. C'est un point important, puisque, en dépit de la persistance de discussions sur les différentes périodes critiques chez les poissons dans leur milieu naturel (Blaxter, 1988), les conditions trophiques à ce stade du développement larvaire demeurent, comme le suggèrent les travaux de Hewit et al. (1985), capitales pour la survie de l'individu.

Gutiérrez E., B. Morales-Nin 1986. Time series analysis of daily growth in Dicentrarchus labrax L. otoliths. J. Exp. Mar. Biol. Ecol. 103, 163-179.

Hewit R.P., G.H. Theilacker, N.C.H. Lo 1985. Causes of mortality in young jack mackerel. Mar. Ecol. Prog. Ser. $26,1-10$.

Karsten U., A. Wollenberger 1972. Determination of DNA and RNA in homogenized cells and tissues by surface fluorometry. Anal. Biochem. 46, 135-148.

Karsten U., A. Wollenberger 1977. Improvements in the ethidium bromide method for direct fluorometric estimation of DNA and RNA in cell and tissuc homogenates. Anal. Biochem. 77, 464-470.

Kendall A.W. Jr., E.H. Ahlstrom, H.G. Moser 1984. Early life history stages of fishes and their characters. In : Ontogeny and systematics of fishes. American Society of Ichthyologists and Herpetologists ed. Special Publication $\mathrm{n}^{\circ} 1,11-22$.

Le Pecq J.B., C. Paoletti 1966. A new fluorometric method for RNA and DNA determination. Anal. Biochem. 17, 100-107.

Regner S., J. Dulčić 1994. Growth of sea bass, Dicentrarchus labrax, larval and juvenile stages and their otoliths under quasi-steady temperature conditions. Mar. Biol. 119, 169-177. 